\title{
Funcionamiento cognoscitivo en la diabetes tipo 2: una revisión
}

\author{
Karina Cerezo Huerta, ${ }^{1}$ Guillermina Yáñez Téllez, ${ }^{2}$ Carlos Alberto Aguilar Salinas, ${ }^{3}$ \\ Juan Manuel Mancilla Díaz ${ }^{4}$
}

Actualización por temas

\section{SUMMARY}

The aim of this work is to offer an updated review of the major cognitive difficulties that appear in Type 2 Diabetes Mellitus (T2DM), and its association with the patient compliance related factors proposed by the Panamerican Health Organization (PAHO), such as characteristics of patient, of the disease and of treatment.

The review included electronic databases search (PubMed, PsycINFO and Springerlink) from January 2000 to December 2011 , predominating research from the United States, Canada, Holland, UK, Japan, Mexico and Germany.

Most of the reviewed articles identified that factors which have shown to be associated with cognitive functioning in T2DM include: glycemic fluctuations, disease duration and pharmacological treatment.

As for the changes that occur in the Central Nervous System (CNS), to date there is no consensus as to whether these are purely degenerative, vascular or a combination of both.

Most affected cognitive abilities are: verbal memory, working memory, verbal fluency, attention, mental planning and psychomotor speed. The latter have been related with frontal, temporal and hyppocampal structure functioning, which are also compromised on T2DM.

In the cognitive aspect, it is reported that T2DM is a risk factor for developing mild cognitive impairment. Also, chronicity of this condition associated with hypertension, inadequate glycemic control, and macrovascular complications increases the odds for vascular dementia to occur

It is concluded that even though some risk factors of the cognitive deficiencies have been identified on T2DM, it is important to determine how they interact, and to what degree they affect overall cognitive performance, and specific functions in this population.

Key words: Type 2 diabetes mellitus, cognitive function, patient compliance.

\section{RESUMEN}

El objetivo de este trabajo es ofrecer una revisión actualizada de las principales dificultades cognoscitivas que se presentan en la Diabetes mellitus tipo 2 (DM2) y su asociación con factores relacionados con la adherencia terapéutica propuestos por la Organización Panamericana de la Salud para las enfermedades crónicas como: las características del paciente, de la enfermedad y del tratamiento.

La revisión se basó en una búsqueda en las bases de datos PubMed, PsycINFO y SpringerLink. Se abarcó el periodo de enero de 2000 hasta diciembre de 2011 , y predominaron las investigaciones procedentes de los Estados Unidos de América, Canadá, Holanda, Inglaterra, el Japón, México y Alemania.

En la mayoría de los estudios consultados se identificó que las fluctuaciones glicémicas, la duración de la enfermedad y el tipo de tratamiento farmacológico son los factores que más se han asociado con el funcionamiento cognoscitivo en la DM2.

En cuanto a los cambios que se producen en el Sistema Nervioso Central (SNC), hasta la fecha no hay consenso en cuanto a si estos son exclusivamente degenerativos, vasculares 0 si son una combinación entre ambos.

En el aspecto cognoscitivo, se ha reportado que la DM2 es un factor de riesgo para desarrollar deterioro cognoscitivo leve y que la cronicidad de esta condición, asociada a hipertensión, a un control glicémico inadecuado y a complicaciones macrovasculares aumenta la posibilidad de desarrollar demencia vascular.

Las habilidades cognoscitivas más referidas como afectadas son memoria verbal y de trabajo, fluidez verbal, atención, planificación mental y velocidad psicomotora, las cuales se han relacionado con el funcionamiento de estructuras frontales, temporales e hipocámpicas, que en la DM2 también se encuentran comprometidas.

Se concluye que a pesar de que se han identificado algunos factores de riesgo de las deficiencias cognoscitivas en la DM2, es importante que se determine cómo interactúan y en qué grado influyen estos factores en el rendimiento cognoscitivo global y en funciones específicas en esta población.

Palabras clave: Diabetes mellitus tipo 2, funcionamiento cognoscitivo, adherencia terapéutica.

Doctorado en Psicología en el área de Neurociencias de la conducta. Universidad Nacional Autónoma de México.

2 Facultad de Estudios Superiores Iztacala. Universidad Nacional Autónoma de México.

3 Departamento de Endocrinología y Metabolismo. Instituto Nacional de Ciencias Médicas y de la Nutrición "Salvador Zubirán". México.

4 Laboratorio de Psicobiología de la Alimentación. Facultad de Estudios Superiores Iztacala. Universidad Nacional Autónoma de México.

Correspondencia: Karina Cerezo Huerta. 4 Oriente 210, La Libertad, 72130. Puebla, Pue. E-mail: kacerez@lapalabra.com; kacerez@yahoo.com.mx

Recibido: 6 de mayo de 2012. Aceptado: 20 de noviembre 2012 


\section{INTRODUCCIÓN}

De acuerdo con la Asociación Estadounidense de Diabetes, ${ }^{1}$ la diabetes mellitus comprende un grupo de enfermedades metabólicas de patogenia multifactorial y poligénica. Estas se caracterizan por la presencia crónica de hiperglucemia, resultado de defectos en la secreción y/o en la acción de la insulina sobre órganos diana, lo que a largo plazo genera repercusiones negativas en varios órganos y sistemas (e.g., ojos, riñones, corazón, sistema circulatorio y sistema nervioso periférico y autónomo).

Este padecimiento se considera un problema de salud pública a nivel mundial, especialmente en los países en vías de desarrollo, ya que de acuerdo con las cifras referidas por la Organización Mundial de la Salud (OMS), actualmente en el mundo existen 346 millones de personas con diabetes y se estima que esta cifra se duplicará para el año $2030 .^{2}$

La diabetes se clasifica en distintos grupos de acuerdo con su momento de aparición y / o tipo de tratamiento -diabetes tipo 1 (DM1), diabetes tipo 2 (DM2), diabetes gestacional y tipos específicos. ${ }^{1}$ En México, de acuerdo con la Secretaría de Salud, ${ }^{3}$ la DM2 es la más frecuente en la población adulta en edad productiva, ya que incluye a $90 \%$ de los casos y resulta ser la consecuencia del envejecimiento de la población y de la adopción de estilos de vida nocivos para el mantenimiento de la salud (e.g. hábitos alimenticios obesogénicos, vida sedentaria y exposición cotidiana a situaciones estresantes).

Con la finalidad de brindar alternativas integrales en la atención que se ofrece a la población diabética, uno de los fenómenos que ha recibido un notable interés desde diferentes áreas del conocimiento es el denominado como adherencia terapéutica, que en esta población generalmente es deficiente, y que de acuerdo con la Organización Panamericana de Salud (OPS) se define como "el grado en que el comportamiento de una persona -tomar el medicamento, seguir un régimen alimenticio y/o ejecutar cambios del modo de vida- se corresponde con las recomendaciones acordadas con un prestador de asistencia sanitaria". ${ }^{4}$

De acuerdo con el organismo mencionado, este fenómeno se divide en cinco factores interrelacionados entre sí: a) los socioeconómicos, b) los relacionados con el equipo de salud, c) los relacionados con la enfermedad, d) los asociados al tratamiento y e) las características del paciente.

Entre los factores mencionados y que se han asociado con mayor frecuencia con el funcionamiento cognoscitivo en la DM2 se encuentran los siguientes: a) las características del paciente (e.g. características demográficas y estado de ánimo), b) los asociados con la enfermedad (e.g. fluctuaciones en los niveles glicémicos, duración, complicaciones y trastornos asociados) y c) con el tratamiento farmacológico (e.g. empleo de agentes orales y/o insulina de manera aislada o combinada). ${ }^{5-15}$

En cuanto a los déficits cognoscitivos que se presentan en la DM2, se ha señalado que éstos son el resultado de fallas en el funcionamiento de estructuras corticales y subcor- ticales (e.g., frontales, temporales e hipocámpicas) e incluyen principalmente fallas en memoria verbal y de trabajo, fluidez verbal, atención, planificación mental y velocidad psicomotora. $^{16-19}$

A continuación se describirán los principales cambios neuroanatómicos que se presentan en la DM2, así como la repercusión que dichos cambios tienen en el funcionamiento cognoscitivo y la asociación que éste tiene con los factores relacionados con la adherencia terapéutica.

\section{CAMBIOS NEUROANATÓMICOS EN LA DM2}

En cuanto al estado del sistema nervioso en la DM2, las dificultades neurológicas descritas con mayor profundidad han sido las relacionadas con alteraciones en el sistema nervioso periférico y/o autónomo (e.g., neuropatías). En cuanto a los cambios en el Sistema Nervioso Central (SNC) y las funciones que regula, hasta la fecha no hay consenso acerca de qué áreas cerebrales se afectan de manera específica y de qué factores intervienen en los cambios cognoscitivos que se presentan.

De acuerdo con Zhao y Halkon, ${ }^{20}$ los mecanismos que regula la insulina a nivel cerebral (e.g., homeostasis energética, plasticidad sináptica y supervivencia neuronal) se encuentran disminuidos en la diabetes, lo que ocasiona que se afecte el metabolismo del $\beta$-amiloide, lo que da lugar a su acumulación en estructuras tanto corticales como subcorticales (e.g., frontales, temporales e hipocampo).

De esta manera, se ha señalado que las alteraciones observadas en el SNC de las personas diabéticas parecen condicionar la aparición de una "encefalopatía diabética", que se presenta con mayor frecuencia en aquellas personas que tienen un control glicémico inadecuado de manera crónica y además presentan complicaciones macrovasculares. ${ }^{21}$

Cabe señalar que aún no es concluyente la evidencia de los cambios mencionados, ya que se ha referido que estos son insidiosos y no resultan estadísticamente significativos cuando se toma como único factor causante a la DM2. Es decir que, debido a que la diabetes suele acompañarse de otros trastornos que se consideran como factores de riesgo cardiovascular (e.g., hipertrigliceridemia, hipertensión, obesidad y aterosclerosis), éstos también pueden contribuir a los cambios mencionados. ${ }^{22,23}$

Mediante el uso de imágenes por resonancia magnética se ha identificado que son dos las alteraciones asociadas con los cambios que presenta el SNC en la DM2 (cuadro 1):

1. Enfermedad cerebral: la DM2 se expresa en forma de lesiones en la sustancia blanca e infartos lacunares, de presentación generalmente asintomática y asociados a la exposición crónica de estados de hiperglucemia y a una larga duración de la enfermedad. ${ }^{17,23}$

2. Degeneración neuronal: que condiciona la presencia de atrofia cerebral predominantemente en regiones fronta- 
Cuadro 1. Hallazgos de neuroimagen con rendimientos cognoscitivos en la DM2

\begin{tabular}{|c|c|c|c|c|c|c|}
\hline No. & Autores & Origen & Diseño & Muestra & $\begin{array}{l}\text { Técnica y/o } \\
\text { instrumento }\end{array}$ & Hallazgos \\
\hline 1 & Den Heijer et al. (2003). & Holanda & Longitudinal & $\begin{array}{l}41 \text { ancianos diabéticos } \\
465 \text { controles }\end{array}$ & $\begin{array}{l}\text { * IRMa } \\
\text { * Listas palabras }\end{array}$ & $\begin{array}{l}\text { La DM2 se asocia con atrofio } \\
\text { hipocampal y amigdalar y es } \\
\text { un factor de riesgo para desa } \\
\text { rrollar DTA }{ }^{b} \text {. }\end{array}$ \\
\hline 2 & Décary et al. (2005). & Canadá & Transversal & $\begin{array}{l}30 \text { ancianos con enfer- } \\
\text { medades crónicas } \\
20 \text { controles }\end{array}$ & $\begin{array}{l}\text { * } \mathrm{EEG}^{\mathrm{c}} \\
\text { * } \mathrm{MMSE}^{\mathrm{d}} \\
\text { * DPRe } \\
\text { * Figura de Rey } \\
\text { * FFS } \\
\text { * Stroop } \\
\text { * TMT ABg }\end{array}$ & $\begin{array}{l}\text { En enfermedades crónicas se } \\
\text { presentan déficits en atención, } \\
\text { memoria de trabajo y diferida } \\
\text { aunado a una actividad lentifi } \\
\text { cada a nivel frontal. }\end{array}$ \\
\hline 3 & Manschot et al. (2006). & Holanda & Transversal & $\begin{array}{l}113 \text { diabéticos } \\
51 \text { controles }\end{array}$ & $\begin{array}{l}\text { * IRMa } \\
\text { * Raven } \\
\text { * TMT ABg } \\
\text { * Stroop } \\
\text { * Figura de Rey } \\
\text { * DCWAIS-III-R }\end{array}$ & $\begin{array}{l}\text { Lesiones en sustancia blanca y } \\
\text { atrofia cortical se asocian con } \\
\text { fallas en velocidad de procesa- } \\
\text { miento y memoria. }\end{array}$ \\
\hline 4 & Brands et al. (2007). & Holanda & Transversal & 40 ancianos diabéticos & $\begin{array}{l}\text { * IRMa } \\
\text { * Raven } \\
\text { * Cubos de Corsi } \\
\text { * RAVLT' } \\
\text { * Figura de Rey } \\
\text { * TMT ABg } \\
\text { * CNTi } \\
\text { * LFTk }\end{array}$ & $\begin{array}{l}\text { Lesiones en sustancia blanca y } \\
\text { atrofia cortical se asocian con } \\
\text { déficits en visoconstrucción. }\end{array}$ \\
\hline 5 & Manschot et al. (2007). & Holanda & Transversal & $\begin{array}{l}122 \text { diabéticos } \\
56 \text { controles }\end{array}$ & $\begin{array}{l}\text { * IRMa } \\
\text { * Raven } \\
\text { * TMT ABg } \\
\text { * Stroop } \\
\text { * Figura de Rey } \\
\text { * DCWAIS-III-R }\end{array}$ & $\begin{array}{l}\text { Asociación modesta entre atro } \\
\text { fia cortical y subcortical con e } \\
\text { rendimiento cognoscitivo. }\end{array}$ \\
\hline 6 & Cervantes et al. (2011). & México & Transversal & 51 diabéticos & $\begin{array}{l}\text { * IRM }{ }^{a} \\
{ }^{*} M^{\prime} M^{d} E^{d} \\
* \text { COGNISTAT }\end{array}$ & $\begin{array}{l}\text { Asociación entre dislipidemia y } \\
\text { enfermedad de pequeño vaso } \\
\text { con fallas en atención, construc } \\
\text { ción y razonamiento analógico. }\end{array}$ \\
\hline 7 & Hayashi et al. (2011) & Japón & Transversal & $\begin{array}{l}61 \text { ancianos diabéticos } \\
53 \text { controles }\end{array}$ & $\begin{array}{l}\text { * } \mathrm{IRM}^{\mathrm{a}} \\
\text { * } \mathrm{MMSE}^{\mathrm{d}} \\
\text { * } \mathrm{HDS}-\mathrm{R}^{1}\end{array}$ & $\begin{array}{l}\text { Asociación entre DM2 con atro } \\
\text { fia hipocampal. }\end{array}$ \\
\hline
\end{tabular}

$a=$ Imagen por Resonancia Magnética, $b=$ Demencia Tipo Alzheimer, $c=$ Electroencefalograma, $d=$ Mini Mental Status Examination, e=Dígitos en Progresión y/o Regresión, f=Fluencia Fonológica y Semántica, $g=$ Trail Making Test A y B, h=Dígitos y Cubos de la Wechsler Adult Intelligence Scale III-R, i=Rey Auditory Verbal Learning Test, $\mathrm{j}=$ Category Naming Test, k= Lexical Fluencia Task, I=Hasegawa Dementia Scale-Review.

les, temporales e hipocámpicas, lo que a su vez se asocia con estados crónicos de microalbuminuria, resistencia a la insulina y a la presencia de hipertensión y complicaciones macrovasculares. ${ }^{16,18,19}$

\section{CARACTERÍSTICAS NEUROPSICOLÓGICAS DE LA DM2}

En estudios transversales y longitudinales se ha señalado que existen cambios cognoscitivos importantes en la DM2 en comparación con poblaciones no diabéticas, dichos cambios se agrupan en los siguientes apartados:
1. La DM2 puede propiciar la presencia de un deterioro cognoscitivo leve, especialmente de tipo amnésico, el cual se exacerba por la presencia de condiciones como una mayor edad y duración de la enfermedad, la exposición crónica a niveles glicémicos inadecuados, complicaciones microvasculares y trastornos asociados (e.g., obesidad e hipertrigliceridemia). ${ }^{24-26}$

2. Se relaciona con el riesgo de desarrollar demencia vascular como resultado de las alteraciones hemodinámicas producidas por la hipoxia e isquemia crónica, cuya presentación es más frecuente en diabéticos ancianos hipertensos con complicaciones macrovasculares. ${ }^{26,27}$ 
Cuadro 2. Asociación entre factores personales y rendimiento cognoscitivo en la DM2

\begin{tabular}{|c|c|c|c|c|c|c|}
\hline No. & Autores & Origen & Diseño & Muestra & Instrumento & Hallazgos \\
\hline 1 & $\begin{array}{l}\text { Arvanitakis et al. } \\
(2006) \text {. }\end{array}$ & EE.UU. & Transversal & $\begin{array}{l}116 \text { ancianos diabéticos } \\
116 \text { controles }\end{array}$ & $\begin{array}{l}\text { * CERAD } \\
\text { * TDB } \\
\text { * DSc } \\
\text { * Stroop }\end{array}$ & $\begin{array}{l}\text { En la DM2 se presentan déficits en } \\
\text { memoria semántica y velocidad } \\
\text { perceptual, asociados al consumo } \\
\text { crónico de tabaco. }\end{array}$ \\
\hline 2 & $\begin{array}{l}\text { Watari et al. } \\
\text { (2006). }\end{array}$ & EE.UU. & Transversal & $\begin{array}{l}40 \text { diabéticos con y sin } \\
\text { depresión } \\
34 \text { controles }\end{array}$ & $\begin{array}{l}\text { * MMSEd } \\
\text { * Stroop } \\
\text { * TMT ABe } \\
\text { * } \mathrm{DS}^{\mathrm{c}} \\
\text { * CVLT } \\
\text { * Figura de Rey } \\
\text { * WCSTg } \\
\text { * FFh } \\
\text { * } \text { FFFTi }^{\mathrm{T}}\end{array}$ & $\begin{array}{l}\text { La asociación de depresión y DM2 } \\
\text { ocasiona mayores déficits de aten- } \\
\text { ción, velocidad psicomotora y fun- } \\
\text { cionamiento ejecutivo. }\end{array}$ \\
\hline 3 & Aberle et al. (2008). & Alemania & Longitudinal & $\begin{array}{l}38 \text { diabéticos } \\
421 \text { controles }\end{array}$ & $\begin{array}{l}\text { * NAli } \\
\text { * FIWAIS-Rk } \\
\text { * } \mathrm{FFh}^{\mathrm{h}}\end{array}$ & $\begin{array}{l}\text { Los cambios cognoscitivos se res- } \\
\text { tringen a los diabéticos ancianos } \\
\text { mayores de } 65 \text { años. }\end{array}$ \\
\hline 4 & Yeung et al. (2009). & Canadá & Transversal & $\begin{array}{l}41 \text { diabéticos } \\
424 \text { controles }\end{array}$ & $\begin{array}{l}\text { * } \mathrm{RVLT}^{1} \\
\text { * } \mathrm{TH}^{\mathrm{m}} \\
\text { * } \mathrm{AEB} \mathrm{B}^{\mathrm{n}} \\
\text { * CTT } \\
\text { * Stroop } \\
\text { * } \mathrm{DS}^{\mathrm{c}}\end{array}$ & $\begin{array}{l}\text { La DM2 aumenta los efectos neu- } \\
\text { robiológicos atribuidos a la edad, } \\
\text { especialmente en velocidad psico- } \\
\text { motora y funcionamiento ejecutivo. }\end{array}$ \\
\hline 5 & $\begin{array}{l}\text { Díaz de León et al. } \\
\text { (2010). }\end{array}$ & México & Longitudinal & $\begin{array}{l}8797 \text { personas mayores } \\
\text { de } 55 \text { años pertenecien- } \\
\text { tes al ENASEMo }\end{array}$ & $\begin{array}{l}\text { *Examen cognos- } \\
\text { citivo transcultural }\end{array}$ & $\begin{array}{l}\text { El funcionamiento cognoscitivo está } \\
\text { determinado por el estado de salud } \\
\text { global y se asocia con un incremen- } \\
\text { to de la mortalidad a dos años. }\end{array}$ \\
\hline 6 & Zihl et al. (2010). & Alemania & Transversal & $\begin{array}{l}28 \text { personas con } \mathrm{DM} 1 \\
12 \text { personas con } \mathrm{DM} 2\end{array}$ & $\begin{array}{l}\text { * TVPp } \\
\text { * LEWMS-Rq } \\
\text { * Raven }\end{array}$ & $\begin{array}{l}\text { Los déficits se presentan en tareas } \\
\text { complejas, cuya naturaleza es pri- } \\
\text { maria y no se pueden considerar } \\
\text { como un efecto secundario de la } \\
\text { sintomatología depresiva. }\end{array}$ \\
\hline
\end{tabular}

a=Consortium to Establish a Registry For Alzheimer's Disease, $b=$ Test de Denominación de Boston, c=Dígito-Símbolo, d=Mini Mental Status Examination, e=Trail Making Test formas A y B, $f=$ California Verbal Learning Test, $g=$ Wisconsin Card Sorting Test, $h=F$ Fluencia Fonológica, $i=$ Ruff Figural Fluency Test, $i=$ Nuremberg

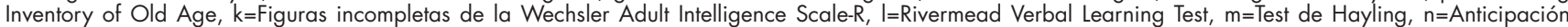
Espacial de Brixton, $\tilde{n}=$ Color Trail Test, o=Estudio Nacional sobre Salud y Envejecimiento en México, p=Tareas de Velocidad Perceptual, q=Localización Espacial de la Wechsler Memory Scale-R.

3. Se ha sugerido que la DM2 es un factor de riesgo que aumenta la posibilidad de que a largo plazo se desarrolle demencia tipo Alzheimer (DTA), especialmente cuando se asocia con complicaciones macrovasculares e hipertensión. Por ello se ha propuesto que posiblemente existe un mecanismo fisiopatológico compartido entre la DM2 y la DTA. ${ }^{28,29}$

\section{ASOCIACIÓN DEL FUNCIONAMIENTO COGNOSCITIVO CON FACTORES RELACIONADOS CON LA ADHERENCIA TERAPÉUTICA}

\section{Factores personales}

\subsection{Estado de ánimo y características demográficas}

Trastornos psicológicos como la depresión y la ansiedad afectan con mayor énfasis a la persona diabética y gene- ralmente se asocian a conductas de adherencia poco efectivas. $^{5}$

Se ha señalado que estos trastornos son más frecuentes en la DM2 y se agravan, especialmente los estados depresivos, cuando se combinan con condiciones propias de la enfermedad (e.g., larga duración, presencia de complicaciones y trastornos asociados), con factores demográficos específicos (e.g., mayor edad, nivel educativo bajo y condiciones socioeconómicas deficientes), con el consumo de sustancias (e.g., alcohol y tabaco) y con un control glicémico deficiente. .5,30,31 $^{2}$

En relación con el rendimiento cognoscitivo se ha señalado que cuando se presentan las condiciones señaladas anteriormente, se corre un mayor riesgo de padecer deterioro cognoscitivo, caracterizado por déficits en tareas de memoria verbal, atención, velocidad de procesamiento y funcionamiento ejecutivo, además de que a largo plazo se asocia con una mayor tasa de mortalidad. 5,30,31

En contraparte, Zihl, Schaaf y Zillmer ${ }^{32}$ argumentan que las fallas en el rendimiento cognoscitivo en la DM2 (es- 
pecíficamente en la memoria de trabajo y tareas que exigen el empleo de habilidades complejas) tienen una naturaleza primaria, y al ser específicas no pueden ser explicadas como un efecto secundario de la sintomatología anímica.

Por otra parte, debido a que en la mayoría de los estudios revisados las muestras estaban conformadas por personas ancianas, se refiere que la edad es una constante que influye en los déficits cognoscitivos presentes en la DM2, especialmente cuando ésta supera los 65 años, siendo las fallas en la velocidad psicomotora y el funcionamiento ejecutivo las más frecuentes. ${ }^{33,34}$

Para consultar con mayor detalle las características de las muestras investigadas y los instrumentos empleados en el grupo de factores personales, en el cuadro 2 se describen las investigaciones mencionadas de manera cronológica.

\section{Factores relacionados con la enfermedad}

\subsection{Fluctuaciones de los niveles glicémicos}

A pesar de que a la DM2 la caracteriza la presencia crónica de hiperglucemia, ocasionalmente puede acompañarse de estados opuestos (hipoglucemia), que, además de influir en la evolución de la enfermedad, también provocan cambios a nivel cognoscitivo. Así, se han realizado varias investigaciones con el objetivo de establecer qué nivel glicémico (hiperglucemia o hipoglucemia) afecta en mayor medida el desempeño cognoscitivo de las personas diabéticas.

De esta manera, se ha observado que los estados crónicos de hiperglucemia, además de favorecer la presencia de complicaciones microvasculares y neuropatías, también provocan efectos bioquímicos tóxicos que aumentan la acumulación de residuos producidos por la glucólisis, generando a su vez daño cerebral isquémico asociado con déficits en la velocidad psicomotora y la atención selectiva. ${ }^{35}$

En el mismo sentido, se ha identificado que cuando la hiperglucemia se presenta de manera aguda en cifras mayores a $300 \mathrm{mg} / \mathrm{dL}$, se incrementa la posibilidad de presentar mayores déficits, en especial en tareas de memoria de trabajo. ${ }^{36}$

Por otra parte, en cuanto a los estados de hipoglucemia, se ha referido que la presencia crónica de dicho estado, además de intensificar la aparición de complicaciones microvasculares a largo plazo, también afecta la precisión en tareas de atención. ${ }^{37}$

En cuanto a las fluctuaciones que se producen en los niveles glicémicos durante el proceso de evaluación neuropsicológica, se ha señalado que, cuando dichos niveles se elevan a lo largo de la evaluación (mayores a $121 \mathrm{mg} / \mathrm{dL}$ ), se producen déficits en velocidad psicomotora y fluencia verbal. ${ }^{38}$

\subsection{Duración, complicaciones y comorbilidad}

Se ha referido que los déficits cognoscitivos que se presentan en la DM2 son resultado de la interacción de la edad, la duración y las complicaciones de la enfermedad, con lo que se afecta con mayor frecuencia la memoria (en especial la verbal), la velocidad psicomotora y el funcionamiento ejecutivo. ${ }^{10,22,24}$

Igualmente se menciona que esta combinación influye negativamente en la fisiopatología del deterioro cognoscitivo presente en la diabetes, debido a que estas condiciones provocan en general un mayor número de infartos cerebrales clínicos y subclínicos, además del incremento y acumulación de $\beta$-amiloide en estructuras cerebrales que regulan el funcionamiento cognoscitivo. ${ }^{20,23}$

Asimismo, se ha referido que cuando la DM2 se asocia con otros padecimientos crónicos (e.g., hipertensión e hipotiroidismo) se presenta una actividad frontal lentificada, lo que se asocia significativamente con deficiencias en atención y memoria de trabajo y verbal inmediata y diferida. ${ }^{39}$

Por otra parte, se afirma que en las personas con DM2 existen "marcadores clínicos" (e.g., hiperinsulinemia, retinopatía y microalbuminuria) que al identificarse sirven como predictores del deterioro cognoscitivo, ya que son un indicador de los efectos degenerativos y vasculares que se producen a nivel cerebral, y que a su vez se asocian con la presencia de fallas en velocidad psicomotora y memoria verbal y de trabajo. ${ }^{40-43}$

Para consultar con mayor detalle las características de las muestras investigadas y los instrumentos empleados en el grupo de factores de la enfermedad descritos, en el cuadro 3 se señalan cronológicamente los estudios mencionados.

\section{Factores relacionados con el tratamiento}

\subsection{Tratamiento farmacológico}

Se ha señalado que si se controla la hiperglucemia y además se logra incrementar la sensibilidad a la recepción de la insulina o bien se promueve su producción residual, posiblemente se pueden reducir los efectos adversos en el funcionamiento cognoscitivo que se presentan en la DM2. ${ }^{14}$

Adicionalmente, se refiere que el deterioro cognoscitivo se presenta en mayor medida en aquellas personas cuyo tratamiento requiere el uso de insulina en comparación con las que utilizan agentes orales o controlan su enfermedad mediante dieta y ejercicio físico. ${ }^{13,35}$

Sin embargo, es importante destacar que debido a que el esquema de tratamiento farmacológico para la persona con DM2 es escalonado y debe tomar en cuenta la evolución del padecimiento, posiblemente el deterioro cognoscitivo sea el resultado de la combinación de la duración, complicaciones y la comorbilidad de la enfermedad, y no sólo del tipo de tratamiento farmacológico prescrito.

Por otra parte, con respecto al tiempo que se requiere para observar cambios en el desempeño cognoscitivo, Shorr et al. ${ }^{14}$ observaron que la monoterapia con agentes orales administrada durante 24 semanas mejoraba considerablemen- 
Cuadro 3. Asociación entre factores de la enfermedad y rendimiento cognoscitivo en la DM2

\begin{tabular}{|c|c|c|c|c|c|c|}
\hline No. & Autores & Origen & Diseño & Muestra & Instrumento & Hallazgos \\
\hline 1 & Ryan y Gekle (2000). & EE.UU. & Transversal & $\begin{array}{l}50 \text { diabéticos } \\
50 \text { controles }\end{array}$ & $\begin{array}{l}\text { * } \mathrm{PA}^{\mathrm{a}} \\
\text { * } \mathrm{DS}^{\mathrm{b}} \\
\text { * } \mathrm{TMT} \mathrm{B}^{\mathrm{c}} \\
\text { * Stroop } \\
\text { * RWAIS-III-R } \\
\text { * TA }\end{array}$ & $\begin{array}{l}\text { El control glicémico deficiente provo- } \\
\text { ca fallas en la velocidad psicomoto- } \\
\text { ra y la atención selectiva. }\end{array}$ \\
\hline 2 & Mc Aulay et al. (2001). & Inglaterra & Transversal & 20 jóvenes voluntarios & * $\mathrm{TEA}^{\mathrm{f}}$ & $\begin{array}{l}\text { Los niveles inducidos de hipogluce- } \\
\text { mia afectan la precisión en tareas } \\
\text { de atención. }\end{array}$ \\
\hline 3 & Hewer et al. (2003). & Alemania & Transversal & $\begin{array}{l}53 \text { diabéticos } \\
29 \text { controles }\end{array}$ & $\begin{array}{l}\text { * }{ }^{\mathrm{TMT}} \mathrm{A}^{\mathrm{c}} \\
{ }^{*} \mathrm{FFSg} \\
{ }^{*} \mathrm{CVLT}{ }^{\mathrm{h}}\end{array}$ & $\begin{array}{l}\text { El restablecimiento glicémico no pro- } \\
\text { duce mejoría sobre el rendimiento } \\
\text { de tareas de fluencia verbal y me- } \\
\text { moria verbal. }\end{array}$ \\
\hline 4 & Hassing et al. (2004). & EE.UU. & Longitudinal & $\begin{array}{l}258 \text { ancianos diabéti- } \\
\text { cos con HASi }\end{array}$ & ${ }^{*}$ MMSEi & $\begin{array}{l}\text { La asociación de DM2 y HASi pro- } \\
\text { vocan mayores déficits cognoscitivos } \\
\text { especialmente después de dos años. }\end{array}$ \\
\hline 5 & Munshi et al. (2006). & EE.UU. & Transversal & 60 ancianos diabéticos & $\begin{array}{l}\text { * MMSEi } \\
\text { * Test del Reloi }\end{array}$ & $\begin{array}{l}\text { El control glicémico inadecuado } \\
\text { provoca déficits en funciones cog- } \\
\text { noscitivas de alto nivel, que afectan } \\
\text { conductas de autocuidado }\end{array}$ \\
\hline 6 & Suzuki et al. (2006). & Japón & Transversal & 13 ancianos diabéticos & $\begin{array}{l}\text { * ADAS-COGk } \\
\text { * DS } \\
\text { * Stroop }\end{array}$ & $\begin{array}{l}\text { La hiperinsulinemia es un marcador } \\
\text { biológico de rendimientos cognosci- } \\
\text { tivos deficientes. }\end{array}$ \\
\hline 7 & Xu et al. (2007). & Suecia & Longitudinal & 1173 diabéticos & * MMSEi & $\begin{array}{l}\text { La asociación de DM2 e hiperten- } \\
\text { sión aumenta el riesgo de padecer } \\
\text { DVI y DTAm. }\end{array}$ \\
\hline 8 & Galanina et al. (2008). & EE.UU. & Transversal & 54 varones diabéticos & $\begin{array}{l}\text { * MMSEi } \\
{ }^{*} \text { COWA }^{n}\end{array}$ & $\begin{array}{l}\text { Al elevarse los niveles glicémicos } \\
\text { (mayores a } 121 \mathrm{mg} / \mathrm{dL} \text { ) se generan } \\
\text { déficits en velocidad psicomotora y } \\
\text { fluencia verbal. }\end{array}$ \\
\hline 9 & lype et al. (2009). & India & Transversal & 71 diabéticos & $\begin{array}{l}\text { * } \text { TMT }^{\mathrm{c}} \\
{ }^{*} \text { CERAD } \\
\text { * } \text { RUDAS }^{\circ}\end{array}$ & $\begin{array}{l}\text { No existe asociación entre las ca- } \\
\text { racterísticas de la enfermedad con } \\
\text { el rendimiento cognoscitivo global. }\end{array}$ \\
\hline 10 & Ishizawa et al. (2010). & Japón & Transversal & $\begin{array}{l}27 \text { varones diabéticos } \\
\text { con y sin sobrepeso } \\
27 \text { controles }\end{array}$ & $\begin{array}{l}\text { * Tareas Go-NoGo } \\
\text { * WCSTp }\end{array}$ & $\begin{array}{l}\text { Las respuestas impulsivas son ma- } \\
\text { yores en pacientes recién diagnosti- } \\
\text { cados, lo que se asocia con niveles } \\
\text { elevados de } \mathrm{HbAlcq} \text {. }\end{array}$ \\
\hline 11 & Shimada et al. (2010). & Japón & Transversal & $\begin{array}{l}103 \text { ancianos diabéti- } \\
\text { cos con y sin DCLr y con } \\
\text { demencia }\end{array}$ & $\begin{array}{l}\text { * MMSEi } \\
{ }^{*} \mathrm{RVLT}^{\mathrm{s}}\end{array}$ & $\begin{array}{l}\text { Los déficits cognoscitivos se asocian } \\
\text { con cifras de } \mathrm{HbAlc} \text { inadecuadas. }\end{array}$ \\
\hline 12 & Grober et al. (2011). & EE.UU. & Transversal & $\begin{array}{l}104 \text { ancianos diabéti- } \\
\text { cos }\end{array}$ & $\begin{array}{l}\text { * FCSRT }{ }^{\dagger} \\
\text { * Series en regresión }\end{array}$ & $\begin{array}{l}\text { El control glicémico inadecuado se } \\
\text { asocia a déficits en memoria y fun- } \\
\text { cionamiento ejecutivo. }\end{array}$ \\
\hline
\end{tabular}

$a=$ Pares Asociados, b=Dígito-Símbolo, c=Trail Making Test A y/o B, d=Rompecabezas de la Wechsler Adult Intelligence III-R, e=Tablero Acanalado, $f=$ Test of

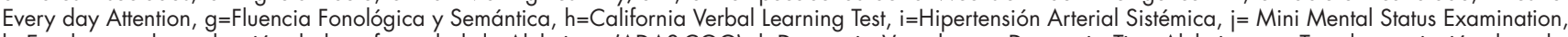
k=Escala para la evaluación de la enfermedad de Alzheimer (ADAS-COG), l=Demencia Vascular, m=Demencia Tipo Alzheimer, $n=$ Test de asociación de palabras COWA, ñ=Consortium to Establish a Registry for Alzheimer's Disease, o=Rowland Universal Dementia Assessment Scale, $\mathrm{p}=$ Wisconsin Card Sorting Test, $q=$ Hemoglobina Glicosilada, $r=$ Deterioro cognoscitivo leve, $s=$ Rivermead Verbal Learning Test, $t=F r e e$ and Cued Selective Reminding Test.

te dicho desempeño, con excepción de tareas en memoria de trabajo, ya que los déficits continuaban presentándose.

\subsection{Régimen alimenticio}

Sin duda alguna, el hábito de una buena alimentación repercute positivamente en la salud general de cualquier individuo y, para el caso de las personas diabéticas, éste resulta ser una condición indispensable para el tratamiento de su enfermedad.

Así, en lo que se refiere a la influencia de la ingesta de carbohidratos sobre el desempeño cognoscitivo en estas personas, se han encontrado déficits en tareas de memoria a corto plazo, en especial después de 30 minutos de haber consumido dichos alimentos, lo que probablemente se aso- 
Funcionamiento cognoscitivo en la diabetes tipo 2

Cuadro 4. Asociación entre factores del tratamiento y rendimiento cognoscitivo en la DM2

\begin{tabular}{|c|c|c|c|c|c|c|}
\hline No. & Autores & Origen & Diseño & Muestra & Instrumento & Hallazgos \\
\hline 1 & $\begin{array}{l}\text { Greenwood et al. } \\
\text { (2003). }\end{array}$ & Canadá & Transversal & 19 diabéticos & $\begin{array}{l}\text { * } \mathrm{MMSE}^{\mathrm{a}} \\
\text { * } \mathrm{RVLT}^{\mathrm{b}} \\
\text { * }{ }^{\mathrm{TMT} \mathrm{B}^{\mathrm{c}}}\end{array}$ & $\begin{array}{l}\text { El consumo de carbohidratos a cor- } \\
\text { to plazo beneficia el rendimiento } \\
\text { cognoscitivo y a largo plazo gene- } \\
\text { ra déficits en memoria. }\end{array}$ \\
\hline 2 & Rosen et al. (2003). & EE.UU. & Transversal & $\begin{array}{l}79 \text { ancianos diabéticos } \\
\text { varones }\end{array}$ & 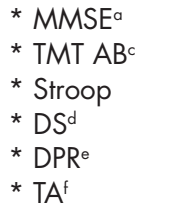 & $\begin{array}{l}\text { No existe un patrón específico de } \\
\text { déficit cognoscitivo asociado a una } \\
\text { pobre adherencia. }\end{array}$ \\
\hline 3 & $\begin{array}{l}\text { Papanikolaou et al. } \\
\text { (2006). }\end{array}$ & Canadá & Transversal & $\begin{array}{l}21 \text { diabéticos con obesi- } \\
\text { dad }\end{array}$ & $\begin{array}{l}\text { * }{ }^{\mathrm{TAVH}} \mathrm{H}^{g} \\
\text { * } \mathrm{TMT} \mathrm{AB}^{\mathrm{c}} \\
\text { * } \mathrm{DR}^{\mathrm{e}}\end{array}$ & $\begin{array}{l}\text { Niveles calóricos altos provocan } \\
\text { déficits en memoria verbal, de tra- } \\
\text { bajo, atención selectiva y funciona- } \\
\text { miento ejecutivo. }\end{array}$ \\
\hline 4 & Ryan et al. (2006). & Inglaterra & Transversal & 145 diabéticos & $\begin{array}{l}\text { * DSd } \\
\text { * } \text { RVLT }^{\mathrm{b}} \\
\text { * CANTAB }\end{array}$ & $\begin{array}{l}\text { Los agentes orales benefician el } \\
\text { rendimiento en tareas de memoria } \\
\text { de trabajo. }\end{array}$ \\
\hline 5 & Roberts et al. (2008). & EE.UU. & Transversal & $\begin{array}{l}290 \text { ancianos diabéticos } \\
1349 \text { controles }\end{array}$ & ${ }^{*} \mathrm{CDRS}^{i}$ & $\begin{array}{l}\text { El tratamiento con insulina y la pre- } \\
\text { sencia de complicaciones macro y } \\
\text { microvasculares se asocian a dete- } \\
\text { rioro cognoscitivo. }\end{array}$ \\
\hline 6 & Devore et al. (2009). & EE.UU. & Longitudinal & $\begin{array}{l}1487 \text { ancianas diabéti- } \\
\text { cas }\end{array}$ & $\begin{array}{l}\text { * } \mathrm{MMSE}^{\mathrm{a}} \\
\text { * } \mathrm{EMBi}^{\mathrm{i}} \\
\text { * } \mathrm{DR}^{\mathrm{e}}\end{array}$ & $\begin{array}{l}\text { El consumo crónico de grasas satu- } \\
\text { radas y transaturadas producen dé- } \\
\text { ficits cognoscitivos en comparación } \\
\text { con el consumo de grasas monosa- } \\
\text { turadas y polisaturadas. }\end{array}$ \\
\hline 7 & Llewellyn et al. (2010). & Inglaterra & Longitudinal & $\begin{array}{l}858 \text { ancianos con y sin } \\
\text { DM2 e HASk }\end{array}$ & $\begin{array}{l}\text { * } \mathrm{MMSE}^{\mathrm{a}} \\
{ }^{*} \mathrm{TMT} \mathrm{AB}^{\mathrm{c}}\end{array}$ & $\begin{array}{l}\text { Los niveles bajos de vitamina } D \text { in- } \\
\text { crementan el riesgo de presentar de- } \\
\text { terioro cognoscitivo a largo plazo. }\end{array}$ \\
\hline
\end{tabular}

$a=$ Mini Mental Status Examination, b=Rivermead Verbal Learning Test, $c=$ Trail Making Test A y/o B, d=Dígito-Símbolo, e=Dígitos en Progresión y/o Regresión, $\mathrm{f}=$ Tablero Acanalado, $\mathrm{g}=$ Test de Aprendizaje Verbal de Hopkins, $\mathrm{h}=$ Cambridge Neuropsychological Test Battery, $\mathrm{i}=$ Clinical Dementia Rating Scale, $\mathrm{j}=$ Escala de Memoria de Boston, k=Hipertensión Arterial Sistémica.

cia con los efectos que se producen sobre el hipocampo y el lóbulo temporal medial. ${ }^{44}$

Por su parte, Papanikolaou et al..$^{45}$ identificaron que el consumo de alimentos con bajos niveles calóricos facilita el desempeño cognoscitivo (memoria verbal, de trabajo y atención sostenida), lo que no sucede con los alimentos con altos niveles calóricos, ya que además de que inducen estados de hiperglucemia repentinos, provocan fallas en tareas de recuerdo diferido.

En cuanto al consumo de alimentos ricos en grasas, se refiere que el consumo crónico (un promedio de seis años) de alimentos con altos contenidos en grasas saturadas y transaturadas, además de que incrementan los niveles de colesterol, a largo plazo se asocia con rendimientos cognoscitivos deficientes, en comparación con los alimentos que contienen grasas monosaturadas, los cuales no afectan dichos rendimientos. ${ }^{46}$

Por otra parte, se ha señalado que en personas ancianas diabéticas e hipertensas que presentan niveles deficientes de vitamina D a largo plazo (durante seis años) se incrementa la posibilidad de presentar deterioro cognoscitivo, ya que el rol preventivo que esta vitamina tiene en la neurodegeneración se encuentra disminuido en estas personas. ${ }^{47}$

\subsection{Ejercicio físico}

La realización cotidiana de este tipo de actividad para la persona diabética resulta ser una de las prácticas prioritarias en su tratamiento integral, ya que uno de sus principales beneficios es el retardo en la aparición temprana de las complicaciones propias de este padecimiento.

Son pocos los estudios que han explorado los efectos que provoca esta actividad en el funcionamiento cognoscitivo. Al respecto Brito, ${ }^{48}$ mediante estudios realizados con roedores, cita que la práctica regular de esta actividad induce cambios neurobiológicos positivos, ya que incrementa la activación neuronal y la irrigación cerebral en el hipocampo, lo que se asocia con mejores rendimientos en tareas de memoria espacial.

Asimismo, se ha referido que cuando las personas diabéticas incorporan la actividad física a su vida cotidiana, además de que reducen la probabilidad de aparición de las complicaciones propias de la enfermedad, también contri- 
buyen a la prevención de los potenciales déficits cognoscitivos que se asocian con este padecimiento. ${ }^{49}$

Igualmente, Indelicato ${ }^{50}$ destaca que, en las personas diabéticas, la memoria de trabajo y la planificación mental son beneficiadas cuando se realiza cotidianamente una actividad física, lo que también se asocia con niveles antropométricos y fisiológicos adecuados (e.g. índice de masa corporal, circunferencia de la cintura y capacidad cardiovascular y pulmonar).

Para consultar con mayor detalle las características de las muestras investigadas y los instrumentos empleados en el grupo de factores del tratamiento, en el cuadro 4 se refieren de manera cronológica los estudios mencionados.

\section{CONCLUSIONES}

La fisiopatología de los cambios del SNC en la DM2 y las respectivas funciones cognoscitivas que regulan las estructuras cerebrales afectadas se atribuyen a fallas en los mecanismos homeostásicos que tiene la insulina a nivel cerebral y a la presencia conjunta de trastornos considerados como factores de riesgo cardiovascular (e.g., hipertensión, hipertrigliceridemia, obesidad y aterosclerosis).

A pesar de que no existe un acuerdo, se sugiere que el efecto combinado de daños vasculares y degenerativos ocasiona alteraciones en el funcionamiento de estructuras corticales (e.g., frontales y temporales) y subcorticales (e.g., hipocampo), lo que a su vez compromete el rendimiento cognoscitivo de estas personas, dando como resultado un deterioro cognoscitivo, expresado en déficits en tareas relacionadas con memoria verbal, de trabajo, fluidez verbal, atención, planificación mental y velocidad psicomotora.

Conforme a la revisión realizada, se puede establecer que dependiendo de qué forma se combinen los factores personales, de la enfermedad y del tratamiento en las personas diabéticas, se presentarán efectos negativos o positivos sobre el funcionamiento cognoscitivo en esta población.

Así, los efectos negativos se presentarán cuando se combinen: a) una larga duración de la enfermedad, b) la presencia de complicaciones y trastornos asociados, c) hábitos alimenticios inadecuados, d) la falta de ejercicio, e) trastornos del estado de ánimo, f) un mal control glicémico de manera crónica, g) una mayor edad y h) un nivel escolar bajo y el consumo crónico de sustancias patógenas (e.g., alcohol y tabaco).

En contraste, al combinarse: a) el mantenimiento de un control glicémico adecuado, b) los hábitos alimenticios favorables, c) la realización cotidiana de ejercicio, d) los estados de ánimo adecuados y e) un nivel escolar elevado, generarán efectos positivos que probablemente favorecerán la conservación de dicho funcionamiento a largo plazo.

Cabe señalar que los hallazgos referidos en cada apartado descrito aún continúan siendo controvertidos, debido a las siguientes cuestiones:
1) Los diseños de investigación en la mayoría de los estudios son transversales y emplean muestras de tamaño reducido, cuyas características clínicas y de tratamiento son heterogéneas.

2) La exploración neuropsicológica de la integridad o deterioro cognoscitivo en esta población se realiza la mayoría de las veces mediante el empleo de instrumentos que evalúan de manera breve el rendimiento cognoscitivo general (e.g., empleo del Mini Mental Status Examination).

3) No existe un consenso con respecto a cuáles son los instrumentos más recomendables para evaluar de manera específica las funciones cognoscitivas más afectadas en este padecimiento. Tampoco existe consenso respecto a en qué momento de la evolución de la enfermedad se debe evaluar con mayor profundidad el rendimiento cognoscitivo de la persona diabética.

En este sentido, es importante que en futuras investigaciones, a pesar de que se realicen estudios transversales, se empleen muestras semejantes por sus características clínicas y de tratamiento, aunado al empleo de grupos control.

Adicionalmente, al explorar la integridad cognoscitiva y su posible relación con factores personales, de la enfermedad y del tratamiento en esta población, además de evaluar el funcionamiento cognoscitivo general, es necesario emplear una batería más compleja de instrumentos para identificar con mayor precisión, y de acuerdo con el momento de evolución de la enfermedad, la influencia de dichos factores sobre funciones cognoscitivas específicas.

De esta manera, a pesar de que se han planteado algunos indicios acerca de la asociación entre el rendimiento cognoscitivo y los factores relacionados con la adherencia terapéutica en la DM2, es importante establecer en qué grado contribuye cada factor, o de qué manera interaccionan para conservar o deteriorar dicho rendimiento, y en qué funciones se presentan los déficits más significativos.

\section{REFERENCIAS}

1. American Diabetes Association. Diagnosis and classification of diabetes mellitus. Diabetes Care 2008;31:62-67.

2. Organización Mundial de la Salud. Estadísticas de la prevalencia de diabetes a nivel mundial. Recuperado en octubre de 2011 http://www. who.int/mediacentre/factsheets/fs312/en/index.html

3. Secretaria de Salud. Numeralia 2008: Diabetes mellitus. Diabetes Hoy 2009;10:2166-2178.

4. Organización Panamericana de la Salud. Adherencia a los tratamientos a largo plazo; pruebas para la acción. Ginebra: OMS; 2004; pp. 1-39, 75-90.

5. Watari K, Letamendi A, Elderkin-Thompson V, Harron E et al. Cognitive function in adults with type 2 diabetes and major depression. Arch Clin Neuropsychol 2006;21:787-796.

6. Munshi M, Grande L, Hayes M, Ayres D et al. Cognitive dysfunction is associated with poor diabetes control in older adults. Diabetes Care 2006;29:1794-1799.

7. Ishizawa L, Kumano H, Sato H, Iwamoto Y. Decreased response inhibition in middle-aged male patients with type 2 diabetes. Biopsychoso Med 2010;4:1-10. 
8. Shimada H, Miki T, Tamura A, Ataka S et al. Neuropsychological status of elderly patients with diabetes mellitus. Diabetes Res Clin Pract 2010;87:224-227.

9. Grober E, Hall Ch, Hahn S, Lipton R. Memory impairment and executive dysfunction are associated with inadequately controlled diabetes in older adults. J Prim Care Community Health 2011;2:229-233.

10. Roberts R, Geda $Y$, Knopman D, Christianson $T$ et al. Duration and severity of diabetes are associated with mild cognitive impairment. Arch Neurol 2008;65:1066-1073.

11. Iype $T$, Shaji $S$, Balakrishnan $A$, Charles $D$ et al. Cognition in type 2 diabetes: association with vascular risk factors, complications of diabetes and depression. Ann Indian Acad Neurol 2009;12:25-27.

12. Hewer W, Mussell M, Rist F, Kulzer B et al. Short-term effects of improved glycemic control on cognitive function in patients with type 2 diabetes. Gerontology 2003;49:86-92.

13. Rosen M, Beauvais J, Rigsby M, Salahi J et al. Neuropsychological correlates of suboptimal adherence to metformin. J Behav Med 2003;4:349-360.

14. Shorr R, de Rekeneire N, Resnick H, Yaffe K et al. Glycemia and cognitive function in older adults using glucose-lowering drugs. J Nutr Health Aging 2006;10:297-301.

15. Ryan Ch, Freed M, Rood J, Cobitz A et al. Improving metabolic control leads to better working memory in adults with type 2 diabetes. Diabetes Care 2006;29:345-351.

16. Den Heijer T, Vermeer S, Van Dijk E, Prins $\mathrm{N}$ et al. Type 2 diabetes and atrophy of medial temporal lobe structures on brain MRI. Diabetologia 2003;46:1604-1610.

17. Manshot S, Brands A, van der Grond J, Kessels $R$ et al. Brain magnetic resonance imaging correlates of impaired cognition in patients with type 2 diabetes. Diabetes 2006;55:1106-1113.

18. Brands A, Biessels G, Kappelle L, De Haan E et al. Cognitive functioning and brain MRI in patients with type 1 and 2 diabetes mellitus: a comparative study. Dement Geriatr Cogn Dis 2007;23:343-350.

19. Hayashi K, Kurioka S, Yamaguchi T, Morita M et al. Association of cognitive dysfunction with hippocampal atrophy in elderly japanese patients with type 2 diabetes. Diabetes Res Clin Pract 2011;94:180-185.

20. Zhao W, Alkon D. Role of insulin and insulin receptor in learning and memory. Mol Cell Endocrinol 2011;177:125-134.

21. Manschot S, Biessels G, de Valk H, Algra A et al. Metabolic and vascular determinants of impaired cognitive performance and abnormalities on brain magnetic resonance imaging in patients with type 2 diabetes. Diabetologia 2007;50:2388-2397.

22. Cukierman T, Gerstein H, Williamson J. Cognitive decline and dementia in diabetes a systematic overview of prospective observational studies. Diabetologia 2005;42:2460-2469.

23. Rajeev K, Jeffrey C, Looi L. Type 2 diabetes mellitus, cognition and brain in aging: A brief review. Indian J Psychiatry 2009;51:35-38.

24. Luchsinger J, Reitz C, Patel B, Tang $M$ et al. Relation of diabetes to mild cognitive impairment. Arch Neurol 2007:64:570-575.

25. Mejía-Arango S, Miguel-Jaimes A, Villa A, Ruiz-Arregui L et al. Deterioro cognoscitivo y factores asociados en adultos mayores en México. Salud Pública Mex 2007;49:475-480.

26. Yaffe K, Kanaya A, Lindquist K, Simonsick E et al. The metabolic síndrome, inflammation, and risk of cognitive decline. J Am Med Assoc 2004;292:2237-2242.

27. Cervantes A, Rodríguez M, Calleja J, Ramírez J. Función cognitiva en pacientes con diabetes mellitus tipo 2: correlación metabólica y por imagen de resonancia magnética. Med Int Mex 2011;27:217-223.

28. Mejía-Arango S, Zúñiga-Gil C. Diabetes mellitus como factor de riesgo de demencia en la población adulta mayor mexicana. Rev Neurol 2001;53:397-405.
29. Xu W, Qiu Ch, Winblad B, Fratiglioni L. The effect of borderline diabetes on the risk of dementia and Alzheimer disease. Diabetes 2007;56:211-215.

30. Díaz de León E, Barrágan A, Gutiérrez H, Cobos H. Desempeño cognoscitivo y mortalidad en personas mayores de 50 años en México. Rev Panam Salud Pública 2010;27:368-375.

31. Arvanitakis $\mathrm{Z}$, Wilson R, Li Y, Thanik $N$ et al. Diabetes and function in different cognitive systems in older individuals without dementia. Diabetes Care 2006;29:560-565.

32. Zihl J, Schaaf L, Zillmer E. The relationship between adult neuropsychological profiles and diabetic patient's glycemic control. Appl Neuropsychol 2010;17:44-51.

33. Aberle I, Kliegel M, Zimprich D. Cognitive development in Youngold type 2 diabetes patients: a longitudinal analysis from the "Interdisciplinary longitudinal study of aging". Curr Psychol 2008;27:6-15.

34. Yeung S, Fischer A, Dixon R. Exploring effects of type 2 diabetes on cognitive functioning in older adults. Neuropsychol 2009;23:1-9.

35. Ryan C, Geckle M. Circumscribed cognitive dysfunction in middleaged adults with type 2 diabetes. Diabetes Care 2000;23:1486-1493.

36. Awad N, Gagnon M, Messier C. The relationship between impaired glucose tolerance, type 2 diabetes and cognitive function. J Clin Exp Neuropsychol 2004;26:1044-1080.

37. McAulay V, Deary I, Ferguson S, Frier B. Acute hypoglycemia in humans causes attentional dysfunction while nonverbal intelligence is preserved. Diabetes Care 2001;24:1745-1750.

38. Galanina N, Surampudi V, Ciltea D, Singh S et al. Blood glucose levels before and after cognitive testing in diabetes mellitus. Exp Aging Res 2008;34:152-161.

39. Décary A, Vendette M, Massicotte J, Mathieu A. A preliminary study of the impact of age-related pathologies on cognitive functioning and waking EEG. N Am J Psychol 2005;7:469-480.

40. Bruce D, Davis W, Casey G, Starstein S et al. Predictors of cognitive decline in older individuals with diabetes. Diabetes Care 2008;31:2103-2107.

41. Hassing L, Hofer S, Nilsson S, Berg S et al. Comorbid type 2 diabetes mellitus and hypertension exacerbates cognitive decline: evidence from a longitudinal study. Age Ageing 2004;33:355-361.

42. Ryan C. Diabetes and brain damage: more (or less) than meets the eye? Diabetologia 2006;49:2229-2233.

43. Suzuki M, Umegaki H, Uno T, Oyun $O$ et al. Association between insulin resistance and cognitive function in elderly diabetic patients. Geriatr Gerontol Int 2006;6:254-259.

44. Greenwood C, Kaplan R, Hebblethwaite S. Carbohydrate-induced memory impairment in adults with type 2 diabetes. Diabetes Care 2003;26:1961-1966.

45. Papanikolaou $Y$, Palmer H, Binns $M$, Jenkins D et al. Better cognitive performance following a low-glycaemic-index compared with a highglycaemic-index carbohydrate meal in adults with type 2 diabetes. Diabetologia 2006;49:855-862.

46. Devore E, Stampfer M, Breteler M, Rosner B. Dietary fat intake and cognitive decline in women type 2 diabetes. Diabetes Care 2009;32:635-640.

47. Llewellyn D, Lang I, Langa K, Muniz G et al. Vitamin D and risk of cognitive decline in elderly persons. Arch Intern Med 2010;170:1135-1140.

48. NO Brito G. Exercise and cognitive function: a hypothesis for the association of type II diabetes mellitus and Alzheimer's disease from an evolutionary perspective. Diabetol Metab Syndr 2009;1:1-7.

49. Colberg S, Somma T, Sechrist S. Physical activity participation may offset some of the negative impact of diabetes on cognitive function. J Am Med Dir Assoc 2008;9:434-438.

50. Indelicato J. Association between executive function, physical activity, and physical fitness in people with type 2 diabetes. USA: Northeastern University; 2009.

Artículo sin conflicto de intereses 